\title{
Application of Choquet Integral-Fuzzy Measures for Aggregating Customers' Satisfaction
}

\author{
Lazim Abdullah $\left(\mathbb{D},{ }^{1}\right.$ Noor Azzah Awang, ${ }^{2}$ and Mahmod Othman ${ }^{3}$ \\ ${ }^{1}$ Management Science Research Group, Faculty of Ocean Engineering Technology and Informatics, \\ Universiti Malaysia Terengganu, Kuala Nerus 21030, Malaysia \\ ${ }^{2}$ Faculty of Computer and Mathematical Sciences, Universiti Teknologi MARA, Shah Alam 40450, Selangor, Malaysia \\ ${ }^{3}$ Fundamental and Applied Sciences Department, Universiti Teknologi PETRONAS, Seri Iskandar 32610, Perak, Malaysia \\ Correspondence should be addressed to Lazim Abdullah; lazim_m@umt.edu.my
}

Received 15 June 2021; Revised 27 August 2021; Accepted 6 September 2021; Published 22 September 2021

Academic Editor: Katsuhiro Honda

Copyright (c) 2021 Lazim Abdullah et al. This is an open access article distributed under the Creative Commons Attribution License, which permits unrestricted use, distribution, and reproduction in any medium, provided the original work is properly cited.

\begin{abstract}
Choquet integral is a type of aggregation operator that is commonly used to aggregate the interrelated information. Nowadays, this operator has been successfully embedded with fuzzy measures in solving various evaluation problems. Inspired from this new development, this paper aims to introduce a combined Choquet integral-fuzzy measures (CI-FM) operator that uses the Shapley value standard and interaction index to deal with the interactions between elements of information. The proposed operator takes into account not only the importance of elements or their ordered positions but also the interaction among criteria during the evaluation process. A case of customers' satisfaction over two fast restaurants in Malaysia is presented to illustrate the application of the proposed aggregation operator. Based on three customers' satisfaction criteria, restaurant 1 and restaurant 2 received CIFM scores of 0.711011 and 0.704945 , respectively. Interestingly, the criterion "services" constantly received the highest rating across both restaurants. In addition, the proposed aggregation operator successfully identified which restaurant is superior in the eyes of customers. Finally, this study offers some research ideas for the future.
\end{abstract}

\section{Introduction}

Current developments in information processing have increased the need for an efficient information aggregation operator. The interaction between criteria exists in a real multicriteria decision-making analysis. When each decisionmaker provides their individual set of criteria, two or more criteria might be redundant and complementary [1]. The Choquet integral is one of the aggregation operators that is used to deal with the interactions between criteria. The intensity of this aggregation operator is reflected by the final Choquet integrated values after performing a series of computations. It was introduced by Choquet [2] with the purpose to solve the interrelationship among criteria of decision problems of which the final ordering of criteria is established. However, ordering of criteria is not a straightforward process as there exists some extent of uncertainty regarding the criteria. Considering this difficulty, the notion of fuzzy measure is used in Choquet integral where interaction phenomena among the criteria can be modelled [3]. The Choquet integral employs the notion of fuzzy measure to indicate the weights or the importance of multiple interdependent criteria in decision-making [4]. In other words, the Choquet integral uses the concept of fuzzy measure to quantify the importance of multiple interdependent criteria. The amalgamation of Choquet integrals and fuzzy measure is further emphasized by $\mathrm{Vu}$ et al. [5]. They reaffirmed that Choquet integral is an aggregating function defined in terms of the fuzzy measure. According to them, a fuzzy measure is a set function that acts on the domain of all conceivable amalgamations of a set of criteria. The Choquet integral aggregation function with fuzzy measure is written as the Choquet integral-fuzzy measure (CI-FM) and will be used throughout this paper. The 
compatibility of this combination may be seen in the way that fuzzy measure permits the Choquet integral to allocate priority to all feasible groupings of criteria in decision problems, allowing for much more aggregation flexibility.

The intricacy of the CI-FM integral lies on the exponential of $2 n$ subsets, where $n$ is the number of criteria in a decision problem [6]. The combination of fuzzy measures and Choquet integrals would result in a thorough aggregation method. The unit interval $[0,1]$ is used to define both the inputs and outputs of CI-FM. However, other intervals are also possible depending on the researchers' preferences. The CI-FM encapsulates the nonadditive capacity property and correlates to a vast class of aggregation functions, allowing greater flexibility in the decision-making process $[7,8]$. Subadditive or superadditive operators are used to integrate functions with respect to the fuzzy measures where many extensions and generalizations of fuzzy sets could be inserted into fuzzy measures. Groes et al., [9] stated that Choquet integral plays a key role for recent advances in the decision theory that encompasses nonadditive measures.

From the above literature, it is clearly seen that the main application of CI-FM can be explained in the decision theory and decision problems. According to Heilpern, [10] the CIFM can be applied to many real decision-making cases in economics and business. It is also reported that CI-FM has been used in specific areas such as insurance [11], green building [12], airline services [13], business education evaluation [14], loan market matching [15], and financial risk [16]. In customer service-oriented research, Pasrija and Srivastava [17] investigated the software quality using the CI-FM. They hypothesized that the CI-FM would be effective in comparing the software solutions that would be executed. In their study, companies and clients need to make appropriate selections on which option to operate with a highquality viewpoint of the ranking. These quality criteria, which are based on multiple points of view, are interdependent and ambiguous in nature. To evaluate user satisfaction, the theory of fuzzy set with a quality model was employed to estimate the software quality. In another customer services research, Vu et al., [5] examined customer preference using the CI-FM. The aims of customers' preferences have always been to support the strategic planning and decision-making of business managers. In their study, the CI-FM has been employed as an aggregation function technique, and they also developed a new toolbox to facilitate the computations. The CI-FM and toolbox have been used to bring advantages to researchers and managers worldwide in performing more effective business and knowledge management decision.

From customers' services research, it is now turning to consumer related research in which goods, products, or services are primarily employed to meet the needs and satisfactions of customers. Oni and Matiza [18], for example, established the value of CI-FM of the main factors that influenced rural-consumer choice of fast-food outlets. In their study, a quantitative survey was conducted, and the number of sample data was collected from two hundred over respondents. The study further investigated that the traditional main factors, such as the value of money, accessibility, and convenience were significantly influenced their choice of fast-food outlets. The survey also identified factors like the taste of food, nutritional value, and variety of menu that were not significant to the choice made by rural consumers. On the other hand, Yan et al., [19] conducted a Kansei evaluation on commercial products, which is an individual subjective judgement of a product's aesthetic appeal. In this evaluation, a three-phase group with no additive multiattribute Kansei evaluation model was proposed. After generating Kansei profiles, a target-oriented Kansei assessment function was presented to induce the Kansei satisfaction utility based on the consumer's personal Kansei preference. In the third phase, an evaluation function was proposed based on the Kansei assessment function and the Choquet integral. On a subset of Kansei characteristics, this entropy-based technique was used to estimate the fuzzy measure.

In another customers' satisfaction research, Peláez, et al. [20] proposed a new purchase decision prediction model in investigating the rank of consumer purchasing factors in digital ecosystem. Evaluations of alternatives and criteria were made by considering the opinions expressed by consumers in digital ecosystems. The opinions expressed are the manifestations of customers' satisfactions over the products, and this is the only input information that is available to the evaluation model. The suggested method discovers the implicit synergies within criteria and alternatives by extracting their weights. Finally, the model uses the CI-FM to suggest aggregation values which eventually determines a purchase ranking. The CI-FM is a tool for describing the correlations between consumers' satisfaction and criteria of multiple products. The advantage of the CI-FM is mostly due to the use of integral and fuzzy measure in its calculations. This computation allows to comprehensively reflect the interaction between all possible combination of criteria in consumers' satisfaction decision problem where information about multiple products and their multiple interdependent criteria are aggregated. Motivated by this advantage, this paper extends the application of CI-FM to another real case study of consumers' satisfaction decision problem. Specifically, this paper aims to propose the Choquet integrated values for two fast-food restaurants in Terengganu Malaysia using the CI-FM. These values are used to suggest the preference of consumers over the criteria and also the more preferred fast-food restaurant over the other. To the best of authors knowledge, this is the first identifiable work of CIFM application to customers' satisfaction. The basic concepts of the fuzzy measures, Choquet integral, and Shapley value standard are recalled in the following section.

\section{Preliminary}

In this section, mathematical definitions of the fuzzy measures and fuzzy integrals are presented. These two definitions are required to understand the whole computational procedures that are encompassed in this paper.

The fuzzy measure is an important tool of aggregating information that is characterized by vague and uncertainty. There are two types of fuzzy measures which are additive and 
nonadditive. If the fuzzy measure is additive, this particular fuzzy measure is termed as the $\lambda$-fuzzy measure. These two types of $\lambda$-fuzzy measures are defined as follows.

Definition 1 (see $[3,21]$ ). Fuzzy measures with $\lambda=0$

Let a universal set $X=\left\{x_{1}, x_{2}, x_{3}, \ldots, x_{n}\right\}$ and $g(x)$ be the worth of all possible subsets. A fuzzy measure $g$ on the set of criteria $X$ is a set function $g(x) \longrightarrow[0,1]$, satisfying the boundary and monotonic properties:

(i) $g(\varphi)=0, g(X)=1$ (boundary)

(ii) If $A \subseteq B \subseteq X$, then $g(A) \leq g(B) \leq g(X)$ (monotonic)

A fuzzy measure is additive if $g(A \cap B)=0$ (disjoints), then the union of two sets can be written as $g(A \cup B)=g(A)+g(B)$. This particular fuzzy measure is termed as $\lambda$-fuzzy measure, a special kind of fuzzy measure defined on $g(X)$ and satisfying the finite $\lambda$-rule. This fuzzy measure is also known as Sugeno measure in which the following additional property is satisfied:

$$
g(A \cup B)=g(A)+g(B)+\lambda g(A) g(B) .
$$

where $\lambda>-1$ for all $A, B \subseteq g(X)$ and $A \cap B=\varphi$. In (1), $\lambda=0$ indicates that the $\lambda$-fuzzy measure $g$ is an additive fuzzy measure and there is no interaction between $A$ and $B$.

Definition 2 (see $[3,21]$ ). Fuzzy measures with $\lambda \neq 0$

If $\lambda \neq 0$, then it indicates that $\lambda$-fuzzy measure $g$ is nonadditive and $A$ and $B$ are interacting with each other. If $\lambda>0$, then $g(A \cup B)>g(A)+g(B)$, which infers that the set $\{A, B\}$ has a multiplicative impact. If $\lambda<0$, then $g(A \cup B)<g(A)+g(B)$, which infers that the set $\{A, B\}$ has a substitutive impact. If $X$ is a finite set, then $\cup_{i=1}^{n} A_{i}=X$. The $\lambda$-fuzzy measure $g$ meets this requirement:

$$
g(X)=g\left(\bigcup_{i=1}^{n} x_{i}\right)= \begin{cases}\frac{1}{\lambda}\left\{\prod_{i=1}^{n}\left[1+\lambda g\left(x_{i}\right)\right]-1\right\}, & \text { if } \lambda \neq 0, \\ \sum_{i=1}^{n} g\left(x_{i}\right), & \text { if } \lambda=0,\end{cases}
$$

where $x_{i} \cap x_{j}=\varnothing$ for all $i, j=1,2,3, \ldots, n$ and $i \neq j$. It can be denoted that $g\left(x_{i}\right)$ for a subset with a single element $x_{i}$ is called fuzzy density and can be written as $g_{i}=g\left(x_{i}\right)$.

The value $\lambda$ may be determined in a unique way from $g(X)=1$, which is equivalent to solve the following equation:

$$
\lambda+1=\prod_{i=1}^{n}\left(1+\lambda g_{i}\right)
$$

Conditions of fuzzy measures are given in Definition 3.

Definition 3 (see [22]). Condition of fuzzy measure

A fuzzy measure on $X$ is a set function $\mu: P(X) \longrightarrow[0,1]$, satisfying the following:

$$
\mu(A \cup B)=\mu(A)+\mu(B)+\lambda \mu(A) \mu(B)
$$

This equation is needed to get fuzzy measure.

If $X$ is finite, then a fuzzy measure parameter $\lambda$ fulfils the following relation:

$$
\mu(X)=\frac{1}{\lambda}\left(\prod_{i=1}^{n}\left(1+\lambda \mu\left(x_{i}\right)\right)-1\right), \quad \lambda \neq 0 .
$$

The parameter $\lambda$ can be determined with the boundary condition $\mu(x)=1$. (i) Boundary condition: $\mu(\varphi)=0$ and $\mu(X)=1$

(ii) Monotonicity: If $A, B \in P(X)$ and $A \subseteq B$, then $\mu(A) \leq \mu(B)$

For $A, B \in P(X)$ with $A \cap B \in \varphi$, the fuzzy measure is said to be as follows:

(i) An additive measure, if $\mu(A \cup B)=\mu(A)+\mu(B)$

(ii) A superadditive measure, if $\mu(A \cup B)>\mu(A)+\mu(B)$

(iii) A subadditive measure if $\mu(A \cup B)<\mu(A)+\mu(B)$

Also,

$\lambda \in[-1, \infty), \forall A, B \in P(X)$ and $A \cap B=\varphi$.

$$
\lambda+1=\prod_{i=1}^{n}\left(1+\lambda \mu\left(x_{i}\right)\right)
$$

Definition 4 (see [2]). Choquet integral

Let $g$ be a fuzzy measure on any set $X$, whose element can be denoted as $x_{1}, x_{2}, \ldots, x_{n}$. The discrete Choquet integral of a function $f: X \longrightarrow R^{+}$with respect to $g$ is defined as follows: 


$$
C_{g}(f)=\sum g\left(A_{i}\right)\left[f\left(x_{(i)}\right)-f\left(x_{(i-1)}\right)\right] .
$$

where $\quad 0 \leq f\left(x_{1}\right) \leq \cdots \leq f\left(x_{n}\right), \quad A_{i}=\left\{x_{i}, \ldots, x_{n}\right\} \quad$ and $f\left(x_{0}\right)=0$. The function $f$ is a permutation of $A_{i}=$ $\left\{x_{i}, \ldots, x_{n}\right\}$.

The Choquet integral and Fuzzy Measures comes with weights of elements. Therefore, the Shapley value standard is used to find weights of fuzzy measures.

Definition 5 (see [23]). Shapley value standard

The Shapley value standard is to identify the fuzzy measure $g_{\lambda}$ as follows:

$$
\operatorname{sh}_{i}\left(g_{\lambda}\right)=w_{i}, \quad \forall i
$$

where $s h_{i}\left(g_{\lambda}\right)$ is the Shapley value of the $i$-th evaluation item of the fuzzy measure $g_{\lambda}$.

The Shapley value can be defined as follows:

$$
s h_{i}\left(g_{\lambda}\right)=\sum_{S \subseteq X} \gamma_{n}(S)[g(s)-g(S) \backslash\{i\}],
$$

where $\gamma_{n}=(n-|S|) !(|S|-1) ! / n !$

This standard makes point of each input's weight, and the weights are always positive values.

These definitions are generally used in the proposed method in which the ultimate decision of the Choquet value could be made based on Definition 4. Detailed explanation of the proposed method is presented in the following section:

\section{Model of Choquet Integral-Fuzzy Measure}

In this section, we define the framework of CI-FM as a tool of the evaluation model. In the proposed framework, there are three main categories in the computation, that is, the inputprocess-output model. Like most of the computation model, the structure of the information processing program are the categories of input, process, and output. In the input category, the evaluation expressed by consumers are assumed as the input data. The true computational procedures are happened in the process category. In this category, fuzzy integrals are the useful instrument for the evaluation. The number of parameters of the fuzzy measure are very large, but the $\lambda$ fuzzy measure is one class of fuzzy measures that is used in this category and suitably identified by interaction index $\lambda$ or $\xi$ and weights of individual evaluation items. In the output category, the CI-FM values are obtained in which the decision of ranking can be established. The CI-FM input-process-output framework can be seen in Figure 1.

To evaluate the fuzzy measure uniquely, we can specify the weights $\left(w_{1}, w_{2}, \ldots, w_{n}, w_{i} \geq 0\right)$ and identification standard. There are three standards that are normally used in the computational process. The standards are a singleton fuzzy measure ration standard, Shapley value standard, and input number standard. In this computation, we use the Shapley value standard to identify the interaction index and fuzzy measure. The detailed computational procedures are proposed as follows:

\section{Proposed Computational Procedures}

In these computational procedures, we adopted the fuzzy measure-Choquet integral calculation system [24] in implementation the computation and finally suggesting the results. The computational procedures are summarized as follows:

Step 1: input $n$ value (number of evaluation items) and $m$ value (number of inputs)

Step 2: insert the names of evaluation items

Step 3: choose the weights identification methods either direct input or pairwise comparison

Step 4: insert the value of the weights

Step 5: choose the interaction degree identification method in which the direct input $\xi \in[0,1]$

Step 6: insert value 0.2 for $\xi$

Step 7: choose the fuzzy measure identification standard (weights' intention) using the Shapley value standard

Step 8: display identified fuzzy measure

Step 9: inputs the value into inputs of Choquet integrals

Step 10: display Choquet integral values

These computational procedures are implemented for the case of customers' satisfaction, and the Choquet integral values are obtained as the final output. These output values would suggest the most preferred evaluation criteria and the more preferred restaurant compared to the other. Detailed implementation of the proposed computational procedures to a consumer satisfaction case study is presented in the following section.

\section{A Case Study}

Customers' satisfaction has long been defined as the provision of goods or services that satisfy the consumers' expectations in terms of price, quality, product, and services [25]. According to Seaw [26], there are three types of basic criteria that need to be observed in most of the studies of customers' satisfaction that related to restaurants. The criteria are food quality, service quality, and venue or location. These criteria are the key factors that influence the customer satisfaction in fast-food restaurants. Qin and Prybutok [27] and Bandara and Gangananda [28] also used these criteria in their research framework where food quality, service quality, and venue are the determinants of customers' satisfaction. With this background, this section describes the case study to evaluate customers' satisfaction over fast-food restaurants using the CI-FM model.

There are two most popular fast-food restaurants operating in the Kuala Terengganu city, Malaysia. Customers were invited to rate the restaurants according to three main criteria in food services in the scale of 1 to 10 to represent from "very dissatisfied" to "very satisfied." The three criteria are food quality, service, and location or venue. In this investigation, we are interested to know the most satisfied criterion out of the three criteria and also which restaurant is better than the other. In meeting this 


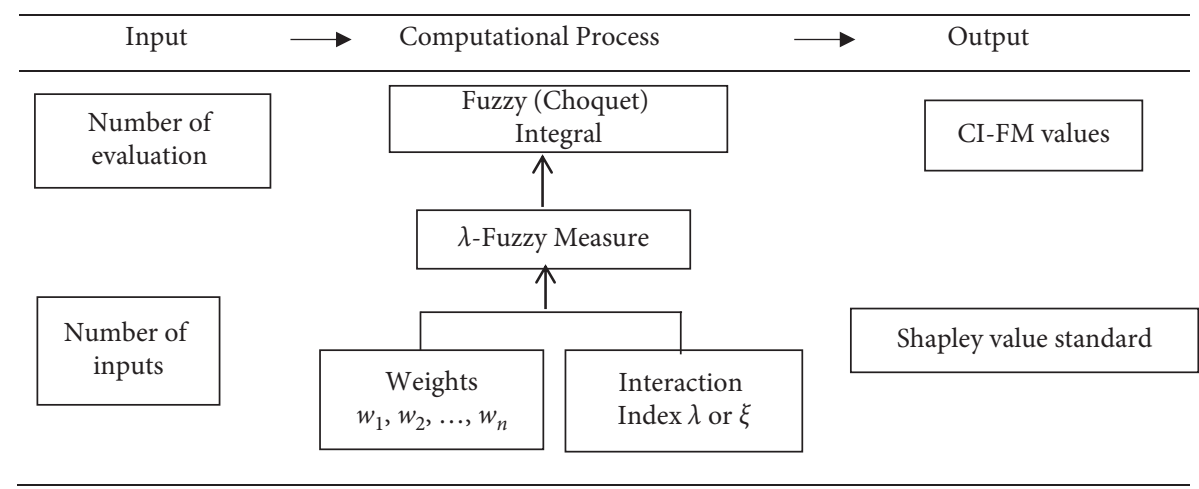

Figure 1: Framework of CI-FM.

purpose, the aggregation operator, the CI-FM is used. Computational procedures of the CI-FM tailored for the case of customers' evaluation are presented as follows.

Step 1. Input 3 value (number of criteria) and 2 value (number of alternatives)

Step 2. Insert criteria; Food, Service and Venue

Step 3. Choose Direct Input as weights identification methods

Step 4. Insert the value of the Weights; 7, 9, 6 respectively.

Step 5. Choose the Direct Input $\xi \in[0,1]$ in the Interaction Degree Identification Method.

Step 6. Insert value 0.2 for $\xi$.

Step 7. Choose the Shapley Value Standard as tool to identify fuzzy measure.

Step 8. Insert the value for each criterion based on the alternatives.

\section{Computational Results}

The output from these procedures is given in Figure 2.

This output text file shows the values of Choquet integrals for restaurant 1 and restaurant 2 in accordance with the respective three criteria.

Step 9. Display Identified Fuzzy Measure

Figure 3 shows the identified fuzzy measures for each set of criteria.

Figure 3 shows the fuzzy measures of each criterion and also the total of fuzzy measures for single criterion, a combination of two criteria, and also a combination of three criteria.

Step 10. Display the FM-CI Values.

Figure 4 displays the final Choquet integrated values for restaurant 1 and restaurant 2 , respectively.
The equation of the Choquet integral is used to be calculate the Choquet value of each alternative. It can be seen that Choquet integrated values for restaurant 1 and restaurant 2 are 0.711011 and 0.704945 , respectively. This finding unveils that restaurant 1 is slightly better than restaurant 2. A brief discussion of the findings and conclusion of this paper are made in the following section.

\section{Discussion}

The major goals of this study are to determine the most important customer satisfaction criterion and to determine which the fast-food restaurant is preferred over the other. The first objective has been achieved where the fuzzy measures of each criterion and their combinations are obtained. This study has shown that the criterion "service quality" gives the highest fuzzy measure of 0.151477 . It is followed by "food quality" with the fuzzy measure of 0.091968 and "venue" with 0.070328 . This result indicates that "service quality" is the most important criterion in the eyes of customers. Service quality is a crucial aspect in the success of fast-food businesses. It is about meeting the customers' satisfactions of what the restaurant has promised. It is also interesting to discuss the findings on the combinations between two criteria. The results reveal that the most important combination is between "food quality" and "service quality" with the value of fuzzy measure of 0.452411 . This is followed by the combination between "service quality" and "venue" with 0.381603 . The least one is the combination between "food quality" and "venue" with 0.259316 . In general, "food quality" can usually relate to how good the meal is prepared, whereas the "service quality" refers to how prompt and courteous the staff are. The findings of this study are now discussed with the findings of previous work. The obtained results are in agreement with Serkan and Serhan's [29] results which showed the strongest indicator of customers' satisfaction is "service quality." Their study surveyed the customer's satisfaction among academicians, staffs, and students at a rural university cafeteria. The article proposes training employees to greet clients politely, to be attentive and cheerful, and to expand their understanding of the food products served to maintain a high level of service quality. In another study conducted by Liu et al. [30], the interrelationships among "service quality," 


\section{INPUTS: Inputs of Choquet Integrals}

\begin{tabular}{|c|c|c|c|}
\hline No. & Food & Service & $\begin{array}{l}\text { Venue } \\
\end{array}$ \\
\hline 1 & 0.7104 & 0.688 & 0.955 \\
\hline 2 & $\mid 0.712$ & $\sqrt{0.6832}$ & 0.915 \\
\hline
\end{tabular}

OUTPUT : TEXT FILES

SUBMIT RESET

FIgURE 2: Output of Choquet integral.

\section{Confirmation: Identified Fuzzy Measure}

Identified Fuzzy Measure
\begin{tabular}{|l|l|}
\hline \multicolumn{1}{|c|}{ Sets } & Fuzzy Measure \\
\hline \hline\{\} & 0 \\
\hline \hline$\{$ Food $\}$ & 0.0919681 \\
\hline \hline Service $\}$ & 0.151477 \\
\hline \hline Food,Service $\}$ & 0.452411 \\
\hline$\{$ Venue $\}$ & 0.0703285 \\
\hline \{Food,Venue $\}$ & 0.259316 \\
\hline \{Service,Venue $\}$ & 0.381603 \\
\hline \{Food,Service,Venue $\}$ & 1 \\
\hline \hline
\end{tabular}

FIgURe 3: Fuzzy measure for each subset.

"customer satisfaction," and "customer loyalty" are examined. The findings of our study also corroborate the ideas of Liu et al. [30] who suggested that the "service quality" positively influences the customers' satisfaction. The study concludes that managers must ensure that they have efficient "service quality" to improve "customer satisfaction" and "customer loyalty" levels.

However, the findings of the current study do not support the previous research conducted by Shamsudin et al. [31] where "service quality" is not significantly contributed to customers' satisfaction. They found that "food quality" is the most important criterion as the customers' are concerned about their health issues such as obesity and diabetes.
The current research findings are also not consistent with the findings of the research conducted by Chun and NyamOchir [32]. They found that food quality and three other criteria of a restaurant positively influence customers' satisfaction. The second purpose of this study has also been accomplished, which is to discover which the fast-food restaurant is preferred over the other. This study suggests that restaurant 1 is preferred over restaurant 2 owing to the higher the Choquet integrated value. It is difficult to explain this result, but it might be related to customers' preference in which customers' preference toward a restaurant is very much influenced by customers' satisfaction. A study indicated that customers' satisfaction has a positive influence on 


\title{
Display: Choquet Integrated Values
}

\author{
Display: Input Values and those Choquet Integrated Values
}

\begin{tabular}{|l|l|l|l|l||}
\hline No. & Food & Service & Venue & Choquet Integrated Values \\
\hline \hline 1 & 0.7104 & 0.688 & 0.955 & 0.711011 \\
\hline \hline 2 & 0.712 & 0.6832 & 0.915 & 0.704945 \\
\hline
\end{tabular}

FIgURE 4: Choquet integrated values of two restaurants.

customer revisit intention and likelihood of recommendation [32]. The preferred restaurant also probably has positive criteria such as food quality, service quality, and customers' satisfaction. The association between the preferred restaurant and these criteria is confirmed by Rajput and Gahfoor [33] where the food quality, restaurant service quality, physical environment quality, and customers' satisfaction positively associated with revisit intentions of customers at fast food restaurants.

\section{Conclusions}

The Choquet Integral-Fuzzy Measure (CI-FM) is a model used to build interaction between criteria of decision problems where the CI-FM values can indicate the best alternatives. In this paper, we presented the application of the CI-FM model to suggest the better fast-food restaurant from the perception of consumers' satisfaction. Evaluation with the linguistic "satisfaction" of two fast-food restaurants in Terengganu Malaysia and three criteria was conducted by a group of customers. The results of Choquet values were obtained after executing series of computational procedures. We begin with identifying input values in which the values 7 , 9 , and 6 are inputted to the model to determine the fuzzy measure for each criterion using the Shapley value standard. In this case, the interaction degree $(\xi)=0.2$ is the parameter used. All in all, this study successfully identified "service quality" as the most important criterion and also identified the better fast-food restaurant using the CI-FM model. This study has three main research novelties. First, the CI-FM model is an enhancement of the Choquet integral with fuzzy measure of which this method is applied to evaluate the customers' satisfaction of two fast food restaurants. This application contributes to the suggestion of a better restaurant in terms of their customers satisfaction based on three criteria. Apart from the practical contribution, this study also contributes to computational procedures used. In Step 7 of the computation where fuzzy measures identification standard is computed, this study chooses the Shapley value standard instead of singleton fuzzy measure standard.
This choice is made to ensure the containment of overall importance of each criterion in terms of its contribution to the score of the three criteria. Finally, the novelty of the study lies upon the weight identification method. In this study, a straightforward method of direct input is used. The direct input method reduces the computational load compared to the pairwise comparison method where weights are directly assigned to the computational procedures. However, the results of this paper are subjected to several limitations. This study used the computational procedures that already built in the systems. With some improvements, for example, development of new pseudo codes may improve the versatility of the model and can be reserved for future research. The second limitation is regarding the criteria and alternatives of the case study. It is believed that the number of criteria and alternatives can be scaled up in future research.

\section{Data Availability}

The linguistic data used to support the findings of this study are available from the corresponding author upon request.

\section{Conflicts of Interest}

The authors declare that they have no conflicts of interest for this publication.

\section{Authors' Contributions}

All authors contributed equally to the writing of this manuscript. All authors read and approved the final manuscript.

\section{Acknowledgments}

The authors thank the Centre of Research and Innovation Management of University Malaysia Terengganu for providing excellent research and academic environments. This study was partly funded by the Centre of Research and Innovation Management of University Malaysia Terengganu. 


\section{References}

[1] R. Lourenzutti, R. A. Krohling, and M. Z. Reformat, "Choquet based TOPSIS and TODIM for dynamic and heterogeneous decision making with criteria interaction," Information Sciences, vol. 408, pp. 41-69, 2017.

[2] G. Choquet, "Theory of capacities," Annales de I'Institut Fourier, vol. 5, pp. 131-295, 1953.

[3] M. Sugeno, Theory of fuzzy integrals and its applications, Ph.D. thesis, Tokyo Institute of Technology, Tokyo, Japan, 1974.

[4] N. Çetin Demirel, T. Demirel, M. Deveci, and G. Vardar, "Location selection for underground natural gas storage using Choquet integral," Journal of Natural Gas Science and Engineering, vol. 45, pp. 368-379, 2017.

[5] H. Q. Vu, G. Beliakov, and G. Li, "A Choquet integral toolbox and its application in customer preference analysis," in Data Mining Applications with $R, \mathrm{Z}$. Yanchang and C. Yonghua, Eds., Academic Press, Cambridge, MA, USA, pp. 247-272, 2014.

[6] A. R. Buck, D. T. Anderson, J. M. Keller, T. Wilkin, and M. A. Islam, "A weighted matrix visualization for fuzzy measures and integrals," in Proceedinhgs of the 2020 IEEE International Conference on Fuzzy Systems (FUZZ-IEEE), pp. 1-8, IEEE, Glasgow, UK, 2020.

[7] T. Murofushi and M. Sugeno, "Some quantities represented by the Choquet integral," Fuzzy Sets and Systems, vol. 56, no. 2, pp. 229-235, 1993.

[8] A. Bonetti, S. Bortot, M. Fedrizzi, R. A. Marques Pereira, and A. Molinari, "Modelling group processes and effort estimation in project management using the Choquet integral: an MCDM approach," Expert Systems with Applications, vol. 39, no. 18, pp. 13366-13375, 2012.

[9] E. Groes, H. J. Jacobsen, B. Sloth, and T. Tranæs, "Axiomatic characterizations of the Choquet integral," Economic Theory, vol. 12, no. 2, pp. 441-448, 1998.

[10] S. Heilpern, "Using Choquet integral in economics," Statistical Papers, vol. 43, no. 1, pp. 53-73, 2002.

[11] M. Amarante and M. Ghossoub, "Optimal insurance for a minimal expected retention: the case of an ambiguity-seeking insurer," Risks, vol. 4, no. 1, p. 8, 2016.

[12] T. O. Olawumi and D. W. M. Chan, "Application of generalized Choquet fuzzy integral method in the sustainability rating of green buildings based on the BSAM scheme," Sustainable Cities and Society, vol. 61, p. 102147, 2020.

[13] L. Zhang, L. Zhang, P. Zhou, and D. Zhou, "A non-additive multiple criteria analysis method for evaluation of airline service quality," Journal of Air Transport Management, vol. 47, pp. 154-161, 2015.

[14] Z. Liao, H. Liao, M. Tang, A. Al-Barakati, and C. LlopisAlbert, "A Choquet integral-based hesitant fuzzy gained and lost dominance score method for multi-criteria group decision making considering the risk preferences of experts: case study of higher business education evaluation," Information Fusion, vol. 62, pp. 121-133, 2020.

[15] D. Liang, Y. Zhang, and W. Cao, " $q$-rung orthopair fuzzy Choquet integral aggregation and its application in heterogeneous multicriteria two-sided matching decision making," International Journal of Intelligent Systems, vol. 34, no. 12, pp. 3275-3301, 2019.

[16] Y. Cao, "Aggregating multiple classification results using Choquet integral for financial distress early warning," Expert Systems with Applications, vol. 39, no. 2, pp. 1830-1836, 2012.
[17] V. Pasrija and P. R. Srivastava, "Evaluation of software quality using Choquet integral approach," International Journal of Fuzzy System Applications, vol. 3, no. 1, pp. 51-81, 2013.

[18] O. A. Oni and T. Matiza, "Factors influencing consumer choice of fast-food outlet: the case of an American fast-food franchise brand operating in a predominantly rural community," Mediterranean Journal of Social Sciences, vol. 5, no. 20, p. 802, 2014.

[19] H.-B. Yan, V.-N. Huynh, and Y. Nakamori, "A group nonadditive multiattribute consumer-oriented Kansei evaluation model with an application to traditional crafts," Annals of Operations Research, vol. 195, no. 1, pp. 325-354, 2012.

[20] J. I. Peláez, F. E. Cabrera, and L. G. Vargas, "Estimating the importance of consumer purchasing criteria in digital ecosystems," Knowledge-Based Systems, vol. 162, pp. 252-264, 2018.

[21] M. Grabisch, Fuzzy Measures and Integrals: Theory and Application, Physica-Verlag, New York, NY, USA, 2000.

[22] M. Sugeno, "Fuzzy measures and fuzzy integrals: a survey," in Fuzzy Automata and Decision Processes, pp. 89-102, NorthHolland, Amsterdam, Netherlands, 1977.

[23] R. E. Alvin, The Shapley Value: Essays in Honour of Lloyd S. Shapley, Cambridge University Press, Cambridge, UK, 1988.

[24] E. Takahagi, "Fuzzy measure-choquet integral calculation system," 2005, http://www.isc.senshu-u.ac.jp/ thc0456/ Efuzzyweb/fm11.html.

[25] B. G. Goff, J. S. Boles, D. N. Bellenger, and C. Stojack, "The influence of salesperson selling behaviors on customer satisfaction with products," Journal of Retailing, vol. 73, no. 2, pp. 171-183, 1997.

[26] T. C. Seaw, "Relationship among service quality, food quality, perceived value, customer satisfaction and behavioural intentions in the Malaysian fast-food industry," Dissertation, University of Malaya, Kuala Lumpur, Malaysia, 2010.

[27] H. Qin and V. R. Prybutok, "Service quality, customer satisfaction, and behavioral intentions in fast-food restaurants," International Journal of Quality and Service Sciences, vol. 1, no. 1, pp. 78-95, 2009.

[28] W. M. A. H. Bandara and A. M. N. M. Gangananda, "An empirical study on the venue attributes on customer satisfaction of wedding banquets in Sri Lanka," International Journal of Engineering and Management Research, vol. 11, no. 1, pp. 156-160, 2021.

[29] M. Serhan and C. Serhan, "The impact of food service attributes on customer satisfaction in a rural university campus environment," International Journal of Food Science, vol. 2019, Article ID 2154548, 12 pages, 2019.

[30] W.-K. Liu, Y.-S. Lee, and L.-M. Hung, "The interrelationships among service quality, customer satisfaction, and customer loyalty: examination of the fast-food industry," Journal of Foodservice Business Research, vol. 20, no. 2, pp. 146-162, 2017.

[31] M. F. Shamsudin, M. F. M. Yazid, S. Hasan, and B. Kadir, "Review on service quality in fast food restaurant: customer satisfaction as mediator," Journal of Critical Reviews, vol. 7, no. 16, pp. 997-1006, 2020.

[32] S.-H. Chun and A. Nyam-Ochir, "The effects of fast food restaurant attributes on customer satisfaction, revisit intention, and recommendation using DINESERV scale," Sustainability, vol. 12, no. 18, p. 7435, 2020.

[33] A. Rajput and R. Z. Gahfoor, "Satisfaction and revisit intentions at fast food restaurants," Future Business Journal, vol. 6, no. 1, pp. 1-12, 2020. 